\title{
Product Lifecycle Management in an Open Industry Framework
}

\author{
Shuichi Fukuda \\ Keio University, SDM, 4-1-1, Hiyoshi, Kohoku-ku, Yokohama, Kanagawa, \\ 223-8526, Japan \\ shufukuda@gmail.com \\ Stanford University, Stanford, CA, 94305, USA \\ shufukuda@cdr.stanford.edu
}

\begin{abstract}
The basic concept of "an Open Industry Framework" is proposed. Current industry framework is closed and industries are making their efforts to produce best quality products in their own particular field. This is because current industries developed on the basis of their inventions. But quickly increasing diversification and frequent and extensive changes are calling for an open framework. In this new framework, industries operate on the basis of Parallel Distributed Processing model. They process multiple tasks in parallel among different types of industries. To achieve such a goal, modular production is called for but current ones are not fully integrated between design and manufacturing. We have to develop a module system which integrates design and manufacturing and which can be shared across industries. When such a system is developed, industries will be divided into two groups. One is subsystem suppliers and the other is system integrators. The subsystem here works in the same way as Lego pieces. Such a framework will enable to distribute production activities to local factories according to their capabilities so that it will increase local employment and their buying power. And we do not have to carry heavy final product over a long distance. Thus, an Open Industry Framework will reduce time to market, cost and energy consumption considerably and industries can be more flexible and adaptive and can satisfy the basic needs of customers.
\end{abstract}

Keywords: open Industry framework, parallel distributed processing, different types of industries, modular production, basic needs.

\section{Introduction}

It is pointed out that the current industries are operating too much independently and that much closer collaboration and sometimes their integration are needed to respond to quickly diversifying needs of our customers.

If we note the non-physical nature of knowledge and experience, a great amount of information can be shared among different industrial sectors and far greater reduction in time to market, cost and energy consumption can be achieved.

If we use computer science terms, what we have been pursuing up to now is to introduce concurrent processing, i.e., to process multiple tasks in parallel on a single 
processor, i.e., in a single industry. Concurrent Engineering is a typical example. But if we process these tasks by distributing them among multiple processors, i.e., among different types of industries, then time to solution or in industrial terms, time to market can be greatly reduced. Therefore, we have to change our product development from concurrent processing style to parallel distributed processing one.

Parallel distributed processing (PDP) product development will change our industry framework from the traditional closed style to open one. Knowledge and experience will be an open source for different types of industries.

Such an Open Industry Framework will be composed of two groups: One is intermediate product producing industries and the other is final product producing industries, i.e., subsystem suppliers and system integrators.

System integrators select modules and assemble them into a final product to truly meet the needs and preferences of customers. Subsystem suppliers can produce their intermediate products in mass and they can supply them to many different types of final product industries. And subsystem suppliers can be divided into several tiers according to their capabilities. The developing countries where knowledge and experience are still not enough to produce complex subsystems can produce simpler ones. Such appropriate assignment of roles would help increase local employment and their buying power.

Such intermediate products or subsystems may be compared to Lego pieces. Lego pieces vary only in shape but these subsystems vary in a wide variety of attributes to cover the whole gamut of choices for system integrators.

\section{Modular Production}

To achieve such a goal, we have to introduce modular production. But current modular design or modular manufacturing focus their attention on design or manufacturing alone and they are not too much integrated. We have to develop modules which can be accessed easily either from design or from manufacturing. In short, it must be able to retrieve the best or better modules from any perspective.

This can be expressed in another way. Current industries are operating in a sequential processing manner and if we use computer processing terms, the way they are operating is forward chaining or data- or technology-driven. (Figure 1).

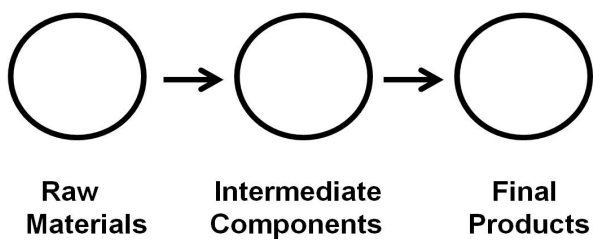

Fig. 1. Current sequential product development

But if we divide industries to subsystem suppliers and system integrators, then system integrators have to find the best or better combination of subsystems easily. Therefore, we have to make our industry framework not only open but also flexible and easily accessible. 
Therefore, modules in an Open Industry Framework must be a versatile tool for many different applications, but their characteristics or features must be clearly identifiable with the specifications of system integrators.

The current industries focus on particular final products and operate independently. But if we focus on intermediate components, there is much common knowledge which can be shared across different industries. Therefore, if we change our focus from final products to intermediate components, then our product development will be such as shown in Figure 2.

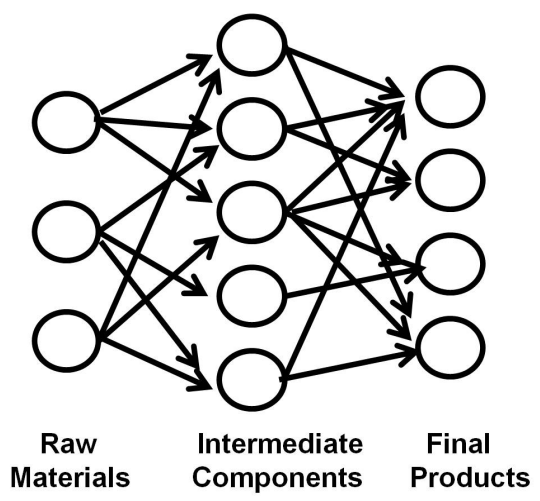

Fig. 2. Parallel Distributed Processing (PDP) Model

We can develop final products by combining different pieces of intermediate components just as we do in playing Lego or to express it in French, in Bricolage style. Lego, however, is just a combination of shapes, but in this case we have to consider many attributes at the same time. But the basic idea is the same as Lego.

This is nothing but a Neural Network. It is interesting to note that a neural network not only represents our brain activities, but it also is analog-based. Such a Neural Network based product development will bring forth greater flexibility and adaptability and allows multi-modal or cross-modal product developments. If we recall a Neural Network used to be called Parallel Distributed Processing (PDP), it will be easily understood that greater reduction of time, cost and energy consumption and greater increase of productivity can be expected.

Modules in the traditional industry framework focused on efficiency but modules in an Open Industry Framework focus on interchangeability or wide applicability. Therefore, modules in an open industry framework also work better for MRO (Maintenance, Repair and Overhaul/Operations) because we can collect data more precisely module by module so that they facilitate introduction of PHM (Prognostics and Health Management) system and makes system health management easier.

\section{Supplier Relationship Management}

Current SRM (Supplier Relationship Management) focuses on relations among a group of suppliers to one industry. It discusses how a tree structure composed of a 
group of suppliers at the lower nodes and a final product industry or user at the top node can work well. The "Open Supplier Relationship Management" discussed here is a network.

\section{$4 \quad$ Basic Needs}

Another important point which must be stressed here is the importance of looking back to the basic needs of customers rather than to keep on going forward on the track of past history of inventions [1], [2]

The Industrial Revolution brought about specialization of industries and current industries developed based upon their own history of inventions. In old days, most of the enterprises are more or less jack-of-all-trades. We have to make our industries more flexible and adaptive.

Take transportation industry for example. We have to change vehicles for land, air and water, because car, airplane and ship industries developed by expanding the technologies invented for a particular transportation.

But if we come back to our basic needs, we would immediately realize what we want is to travel comfortably and without troubles. No one wants to change vehicles. We do so because we have to. Thus, most of current industries are technology-driven. They say customers' voices are important. But these customers mean the users of their products developed by their own technologies [3].

We have to note that B to B relations are nothing other than $\mathrm{S}$ to $\mathrm{C}$ (Supplier to Customer) relations. Customers, no matter whether they are end-users or business enterprises, would like to select industries as they like in order to meet their needs and preferences. However, customers have no other choices than to select among the samples offered by the final product industries. They are supposed to be passive consumers.

But customers have their dreams and they would like to make their dreams come true. If we look back into the very old days, man produced tools to realize their dreams. Tools are nothing but tools, but what man really wanted was to make his dream come true. Why man is called Home Faber is because he makes tools to realize their dreams. Animals can use natural objects as tools. But they do not manufacture tools to realize their dreams. Man can look into the future. But animals live for now [4]. Homo Faber is not trying to develop a better tool, but what he is trying is to make his dream come true and in order to realize that, he produces a tool.

But current industries are making efforts to produce better tools and seem to forget why they are offering such tools or what their customers really want.

Let us consider Brazil. She is so big that there are air taxis. But even if you can use an air taxi and you can land where you want, you cannot go any further, if a car is not available there. So integration of airplane and car is a necessity in Brazil. Such requirements vary from country to country, from region to region and from person to person. What is important is to satisfy such basic needs or requirements. They are not asking us to produce a better airplane. 
Thus, we have to re-organize industries to satisfy the true needs or demands of our customers. We have been discussing how we can meet diversifying needs, but these discussions are focused on one particular industry and more often than not with particular final product in mind. We have not really look back to the basic needs of our customers. Our final goal is to make their dreams come true. It is not to provide our customers with the best quality products or tools.

\section{Integration of Design and Manufacturing into a Module}

Then, how can we make our industry framework flexible and adaptive enough to meet such basic requirements. As discussed before, it is to introduce modular design and manufacturing. But current modular design and modular manufacturing are developed separately and not integrated enough and what is most lacking is that the current product development is forward chaining or technology driven. If we are going to realize such an Open Industry Framework, we have to change it so that it also enables backward chaining or needs- or goal-driven processing. Or we should say we have to change it from one way to both ways. Then, how can we integrate design and manufacturing together into a module to achieve such a goal?

\section{$6 \quad$ An Illustrative Example}

Let us take AWS (American Welding Society) standard for example. AWS standardizes welding procedures in terms of system components or sub-assemblies such as a box for example (Figure 3).

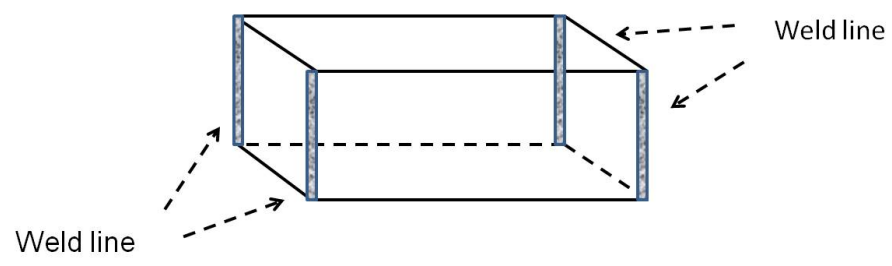

Fig. 3. AWS Standard

Ship design is fundamentally based on boxes and boxes are used in many other areas such as cars, trains, ships, houses, containers, etc. If we introduce such a box type design, it would facilitate integrating car, plane and ships into one. This standard is for welding, but such standardization would greatly facilitate integrating design and manufacturing. And it would make introduction of robots much easier.

And if we use such a method as DSM (Design Structure Matrix) [5] by paying close attention to the integration of design and manufacturing as illustrated here, such modules proposed here will be developed. 


\section{Concurrent Engineering (CE)}

CE proved how effective it is to integrate design and manufacturing knowledge and experience [6], [7]. The success of CE can be attributed to the fact that it noted the nonphysical nature of our knowledge. Since knowledge and experience are non-physical so we can pack them in a much smaller box than physical elements (Figure 4).

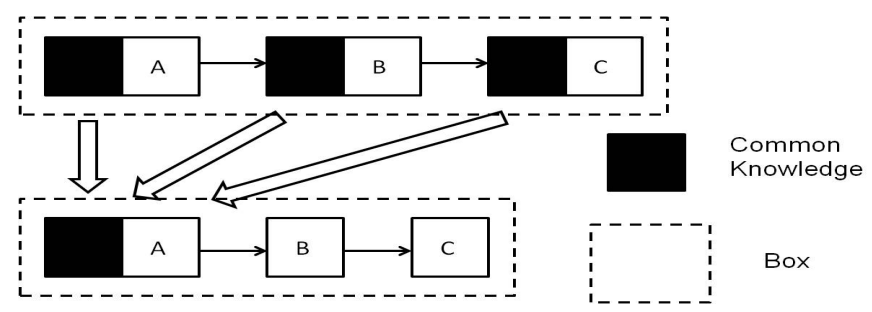

Fig. 4. Concurrent Engineering as a packing problem

\subsection{Traditional CE: Reduction of Time}

Traditional CE focused its attention on how we can reduce time to market because it was originally developed for military competition, although later it was expanded to industry sectors. Thus, reduction of time to realize a product was mandatory. Reduction of time to market is also welcomed by industry, because they are fighting another war on the market. And this reduction meant nothing other than increase of productivity. Increasing productivity was becoming more and more difficult. But if we note knowledge is non-physical, we were able to increase productivity remarkably. Thus, CE spread very quickly and widely.

The advantage of CE is not reduction of time to market alone. DARPA Initiative in Concurrent Engineering (DICE) project [8] spreads the name of CE, but what DICE tried to achieve is to develop a competitive weapon, when you find out your enemy is developing a better weapon. Then, you have to develop a equally capable weapon until your enemy realize it. So reduction of time to realization is crucial. But we have to remember that we have to achieve this goal with the current resources. So how we can make our product development flexible and adaptive enough with current resources was what CE pursued.

The idea of CE presumably comes from Concurrent Processing (CP) in Computer Science. What we should note is that $\mathrm{CP}$ is to process multiple tasks in parallel, but on a single processor. If we regard one industry as one processor, $\mathrm{CE}$ is exactly the same as $\mathrm{CP}$.

\subsection{New Role of CE: Reduction of Energy}

But today CE is not so much appreciated by industry as it was. Why? This is because customers' requirements are diversifying very quickly and extensively today. Yesterday, industry could survive by mass production. So CE yesterday had much to offer 
for industry. But today industry has to customize their products to meet the diverse requirements of their customers.

Another emerging issue of importance is the lack of energy. Industry has to reduce energy consumption. But we should remember energy reduction is very important for industry from the first. Energy saving is important for keeping the world green, but apart from that, reduction of energy consumption is nothing other than cost reduction. The more we can reduce cost by reducing energy consumption, the more profit we can obtain. Then, how can we adapt CE to such changing situations?

\subsection{Expanding CE into 2 Dimensions of Time and Space}

If we recall that $\mathrm{CE}$ solved a packing problem with attention paid to the non-physical nature of knowledge, we could find our solution. CE yesterday only solved one dimensional problem. It solved the problem with respect to time. But if we expand CE into 2 dimensions, i.e. time and space, then we can adapt $\mathrm{CE}$ to the current situations.

Thus, CE today will be illustrated as shown in Figure 6. The core idea of sharing knowledge is the same. In fact, if we expand CE from one dimension to two dimensions, the reduction of space would be greater so that we could pack processes in much smaller box (Figure 5).

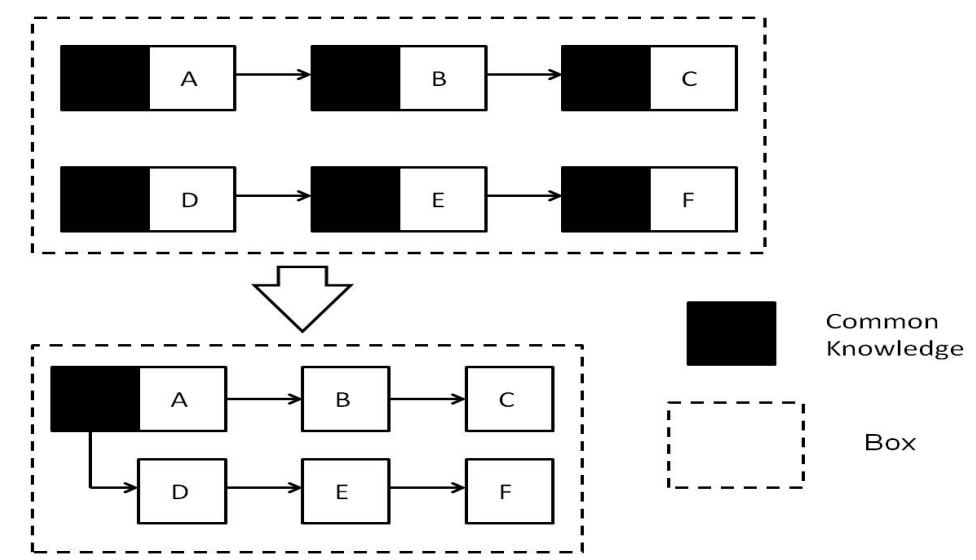

Fig. 5. Concurrent Engineering in two dimensions

\section{From Concurrent Processing to Parallel Distributed Processing}

In CS area, it was soon found out that we can reduce time considerably if we use multiple processors. This leads to Parallel Distributed Processing (PDP) on multiple processors. If we use PDP, then the time to solve can be reduced remarkably.

So if we come back to our original discussion about industry framework, we can reduce time to market very remarkably if we distribute our tasks among multiple 
industries. Of course, reduction of time is nothing other than reduction of cost and energy consumption. So, collaboration among industries will bring forth much greater benefits for those involved.

Modularization of design and manufacturing will expedite such industry-wide collaboration. But such modules should be something like Lego pieces. Current modules focus on efficiency, but they must enable a versatile selection for the customers. Although customers do not select and combine them into a final product by themselves and system integrators carry out such jobs for them, the recent remarkable progress of digital manufacturing such as 3D printers, etc, will visualize customers' dreams more easily and clearly so the integrators can understand what kind of products they really want and integrators can work together with them to find appropriate elements and combine them into the final product their customers expect.

\section{Benefits of an Open Industry Framework}

Integrated design and manufacturing subsystem standardization will lead to true glocalization. Components can be purchased from factories near the market and assembled locally. Local people can assemble them into a final product to meet their needs and to their preferences. And it will reduce the necessity to transport heavy final products over a long distance. If some components cannot be procured near the market, we can import them from nearby countries or regions. The energy, time and trouble to transport such components to the market can be minimized.

Current economy is centralized. But such component standardization in design and manufacturing across industries will open a door to autonomous distributed economy system. Then, far greater reduction of energy and much improved productivity will be achieved. Furthermore, it will meet local people's needs to a T and bring much greater satisfaction to them.

And it should also be stressed that final product industries do not have to stick to particular types of final products as they are doing now. What they need is an ability to integrate various elements into a final product so they can be very flexible and adaptive. They do not have to worry about their stocks any more. Subsystem suppliers are happy too. They do not have to worry because they can supply their subsystems to far wider range of integrators or customers.

Therefore, an Open Industry Framework proposed here will bring forth Win-Win relations for all.

\section{References}

[1] Fukuda, S.: Emotional Engineering, vol. 1. Springer (2011)

[2] Fukuda, S.: Emotional Engineering, vol. 2. Springer (2013)

[3] Fukuda, S.: Concurrent Engineering in a New Perspective: Heading for Seamless Engineering. In: Stjepandic, J., Rock, G., Bil, C. (eds.) Concurrent Engineering Approaches for Sustainable Product Development in a Multi-Disciplinary Environment, vol. 1, pp. 15-26. Springer (2013) 
[4] What Will Our Consciousness about the Future, Which is Specific to Human Bring to Us? In: Applied Brain Science Symposium, Institute of Science of Survival (2010) (in Japanese)

[5] Eppinger, S.D., Browning, T.R.: Design Structure Matrix Methods and Applications (Engineering Systems). MIT Press (2012)

[6] Sriram, R., Logcher, R., Fukuda, S.: Computer-aided Cooperative Product Development. Springer (1991)

[7] Frey, D.D., Fukuda, S., Rock, G. (eds.): Improving Complex Systems Today. Springer (2011)

[8] http://www.dtic.mil/dtic/tr/fulltext/u2/a226394.pdf 